In: Synthese, special issue "Theoretical Frameworks and Empirical Underdetermination". Submitted November 2008, revised March 4, 2009.

\title{
Is Structural Underdetermination Possible?
}

\author{
Holger Lyre ${ }^{1}$
}

\begin{abstract}
$\underline{\text { Abstract }}$
Structural realism is sometimes said to undermine the theory underdetermination (TUD) argument against realism, since, in usual TUD scenarios, the supposed underdetermination concerns the object-like theoretical content but not the structural content. The paper explores the possibility of structural TUD by considering some special cases from modern physics, but also questions the validity of the TUD argument itself. The upshot is that cases of structural TUD cannot be excluded, but that TUD is perhaps not such a terribly serious anti-realistic argument.
\end{abstract}

\section{The Thesis of Theory Underdetermination and its Neighbours}

For some the thesis of theory underdetermination (TUD) belongs to the common wisdom of philosophy of science:

TUD: For any theory $\mathrm{T}$ and any body of observation $\mathrm{O}$ there exists another theory $\mathrm{T}^{*}$, such that $\mathrm{T}$ and $\mathrm{T}^{*}$ are empirically equivalent, but ontologically different.

The central intuition behind TUD is that since theory exceeds observation it is more than plausible to assume that there always exit many, if not infinitely many, rivals to any given theory that satisfy the corresponding body of empirical evidence. The TUD thesis can in particular be found in Quine (1975): "If all observable events can be accounted for in one comprehensive scientific theory - one system of the world... - then we may expect that they can all be accounted for equally in another, conflicting system of the world. We may expect this because of how scientists work. For they do not resist with mere inductive generalizations of their observations: mere extrapolations to observable events from similar observed events. Scientists invent hypotheses that talk of things beyond the reach of observation. The hypotheses are related to observation only by a kind of one-way implication; namely, the events we observe are what a belief in the hypotheses would have led us to expect. These observable consequences of the hypotheses do not, conversely, imply the hypotheses. Surely there are alternative hypothetical substructures that

${ }^{1}$ Philosophy Department, University of Bielefeld, Germany, Email: lyre@uni-bielefeld.de 
would surface in the same observable ways. Such is the doctrine that natural science is empirically under-determined ... by all observable events."

Quine's TUD is a particularly strong thesis, or at least this is how I would like to reconstrue it - to keep the thesis an interesting one and not to mix it with other wellknown topics in the philosophy of science. For TUD shows similarity and conceptual proximity but should not be confused with either the idea of confirmational holism (Duhem or Duhem-Quine thesis) or Hume's problem of induction. Duhemian confirmational holism, at first, is based on the idea that scientific theories can only be confirmed as a whole, or, as a corollary, no scientific hypothesis can be tested in isolation. This can easily be motivated under the (perhaps obsolete but nevertheless still instructive) view of theories as sets of theses and statements. It is possible, or so the Duhem-Quine thesis goes, to adhere to a scientific statement in the face of adverse observations by revising other theses. Only the whole set is amenable to empirical confirmation. Apparently, there's only a small gap to TUD from here: it seems plausible that we may generate rivalling theories simply by readjusting the total system of hypotheses. Note, however, that, given TUD, even the total system is underdetermined by observation - a possibility not considered by conformational holism. Moreover, Hume's induction problem points to some form of underdetermination as well. Such Humean underdetermination can be understood as underdetermination of theory by all past evidence or, even more generally, by any limited evidence, be it temporally, spatially or whatever. TUD, in contrast, involves a stronger claim since it considers the possibility of underdetermination even in the visionary case of all possible observations.

\subsection{TUD and the problem of missing examples}

Thus, TUD is a strong thesis. And if true it is perhaps the most serious attack on scientific realism. Of course: the stronger, the more interesting. On the other hand: the stronger, the more implausible and perhaps even wrong. And, indeed, there are clouds on the horizon for TUD proponents. For TUD suffers from a severe but rarely considered problem of missing examples. One of the few philosophers of science who addresses this problem, if only briefly, is John Earman (1993, p. 31): "Are there interesting cases of empirically indistinguishable theories? ... Here I find the philosophical literature disappointing ... [but] I claim ... that what we have is a shortcoming of the philosophical literature and not a failure of the underdetermination thesis."

Is Earman right? He diagnoses an important problem - and at the same time discards it as "a shortcoming of the philosophical literature." But we should not be convinced so easily. Given the generality of the TUD thesis as it is often stated in the philosophy of science literature one would assume that practising scientists suffer seriously from TUD implications. One would assume that, in their daily work, practising scientists across all fields are forced to spend a lot of their working time on trying to disentangle the always appearing and re-appearing rivalling theories with their contradictory ontological 
implications. One would assume, in fact, that theoretical science, as we know it, is practically almost impossible, or, at least, seriously hindered. But nothing of the sort is the case. On the contrary, practising scientists have usually never heard about the TUD thesis nor spent a minute in their scientific life disturbed by something like it. Science in its practice is not a battlefield of contradictory theories, science is largely conservative and calm.

So, again, given the generality of the TUD thesis, the fact that there do not exist (at least not that many!) interesting examples is indeed a pressing problem for TUD - and we should read Earman as a challenge to philosophy of science to come up with some more interesting examples. So let's not evade but try to face Earman's challenge.

\subsection{Classes of TUD examples}

To be sure, there are TUD examples. The relevant question, however, is whether there is a considerable number of interesting examples, that is cases, where we are confronted in the mature sciences with puzzling TUD scenarios. And I maintain that this is not the case. But some more general considerations first.

We should try to structure the less interesting examples a bit. Here's a possible list of classes of cases: (i) pathological cases, (ii) empirical limitations cases, (iii) conventionalismborderline cases, (iv) historic cases. Let's consider them one after another.

\section{(i) Pathological cases}

Consider a law-like equation $G(X, t)=0$ and another equation $G(X, t)+g(t)=0$, where $g(t)$ $=g_{0}(t)\left(t-t_{1}\right)\left(t-t_{2}\right) \ldots$ with a set of parameters $X$, an arbitrary function $g_{0}(t)$ and measuring time points $t_{i}$. Both equations will satisfy exactly the same set of observations, however, due to the arbitrariness of $g_{o}(t)$ they allow for drastically different ontologies (we could think of $g_{o}(t)$ as an additional force, say). Our world might behave extremely bizarre at any time nobody observes it. This I call a pathological case. ${ }^{2}$ It cannot be ruled out empirically, since it is designed in such a way that it is auto-immune to falsification. A well-known instance of this is naïve creationism, for instance the statement that the entire world (including all records and fossils of the past) has emerged just 5 minutes ago. It is obvious that such cases, albeit logically possible, are not really thrilling. In science we take notice of meta-criteria such as simplicity, economy, coherence in order to single out such pathological, non-falsifiable cases.

\footnotetext{
${ }^{2}$ Laudan and Leplin (1991, p. 463) judge similar cases as `logico-semantic trickery’, while Hoefer and Rosenberg (1994, p. 604) just speak of 'cheap tricks'.
} 
It is not implausible to assume that there exist general boundaries of technical feasibility in our man-made experiments. It might, for instance, for ever be an impossibility to make measurements about the physics at the Planck scale. Such technical boundaries then leave all models, which reach theoretically beyond the boundaries, empirically underdetermined. But who knows what the future will bring. Technological developments might astonish us, as they did in the past, at least sometimes. More tricky cases of apparent underdetermination are considered by Glymour (1977) and Malament (1977), who discuss models of cosmologies where even idealized observers, observers who live forever, are unable to determine the global topology of space. I like to consider both examples as instances of the same type of TUD, call it TUD from empirical limitations. In such cases the underdetermination does not originate from the general fact that theory exceeds evidence but from our particular circumstances as measuring observers in the world. It is not due to an in-principle-matter of how theories relate to empirical data, but due to our limited empirical, observational or technical means that we can practically not decide about the physics at the Planck scale. Hence, it is in principle possible to reconcile such cases with a realistic attitude.

\section{(iii) Conventionalism-borderline cases}

Perhaps the most interesting class of allegedly TUD cases is connected to the issue of spacetime conventionalism. Here the claim is that the choice of geometry to describe spatiotemporal relations is conventional, and that questions about the true geometry of spacetime have no operational meaning. A well-known example is Poincaré's disk, where the option between non-Euclidean geometry versus Euclidean geometry plus systematic deformations of the measuring rods is considered. Another, perhaps more intriguing example is the so-called Hollow earth. Despite the many weird cult stories which can still be found today in connection with it, the Hollow earth picture can be presented in a mathematically straightforward way such that it cannot be refuted on empirical grounds. This can basically be seen from the pure fact that a mere spherical coordinate transformation, the inversion at the sphere $r^{\prime}=R^{2} / r$ (with earth radius $R$ ), mediates between the conventional convex picture of the earth and the concave Hollow earth. Suppose further that light in Hollow earth travels on circles through the center of the Hollow earth, then every physical process or event can be "translated" into the Hollow earth scenario and no experiment can ever distinguish between the two views. ${ }^{3}$

\footnotetext{
${ }^{3}$ Some might complain that a Jules Vernean "Journey to the Center of the Earth" will help settle issues. Note that the center of the hollow Earth corresponds to the infinite point in our traditional convex picture, while the center of our convex earth corresponds to the infinite point in the Hollow earth scenario. If it were possible to travel through the center of the earth and reappear at the antipodal point (in the convex picture of the spherical earth), wouldn't this clearly demonstrate the truth of the convex picture? Wouldn't this provide a nice experimentum crucis? Well, there's always a possibility to circumvent adverse observations: The Hollow earth proponent might just introduce a suitable hypothesis, in this case a wrap-aroundcondition for any measuring rod (or matter wave-function) $Y(r)$ such that $Y(r)=Y(-r)$ in the limit $|r|{ }^{\circledR} 0$. By this move we arrive at an excellent textbook example for Duhemian holism - with Hollow earth still empirically irrefutable.
} 
Now, why call this a conventionalism-borderline case? As Quine puts it (in connection with the almost analogous Poincaré example): "[The] case ... due to Poincaré ... is less simple than the mere switch of 'electron' and 'molecule' [in any textbooks], but it presents no serious challenge. The two formulations are formulations, again, of a single theory" (Quine 1975, p. 322). What he means is that if we can shift back and forth between two views by a mere reconstrual of predicates (under the assumption of formulating theories in predicate logic), then the two formulations aren't formulations of two ontologically distinct theories, but rather two formulations of one and the same theory. The issue, of course, is to have a clear criterion for determining ontology from theory formulations. And this, of course, is a deep and long-discussed problem. Quine himself was a bit undecided about it, since years later we find him with the statement that the Poincaré scenario should just be considered "...drastically unlike theory formulations" (Quine 1990, p. 13). The point is that in Poincaré's example as well as in the Hollow earth example, while it is possible to present a "lexicon" to translate differing predicates (e.g. between convex and concave picture: straight line circle, point at infinite distance - center, center of earth - point at infinite distance), certain overall features of the two views are quite distinct. The Hollow earth, for instance, seems to break the Principle of Homogeneity of Space (as well as, perhaps, the Cosmological Principle), since in it the center of the earth and, in fact, the whole planet earth, becomes a physically distinguished object. Why not have a Hollow Venus or Hollow Alpha Centauri instead? What's so special about earth? Those who find such differences sufficient for an ontological difference, will follow the later, not the earlier Quine and vice versa. I will not delve any further into these issues here, but hope to have said enough to make plausible why one might judge such cases as borderline cases.

\section{(iv) Historic examples of TUD}

The last class in my list can be handled quite quickly and will, at the same time, lead over to the next section. Most examples of TUD cases that can be found in the literature are, in fact, examples from the history of science. Van Fraassen (1980, p. 46), for instance, considers Newton's theory TN (Newtonian mechanics plus gravity) and the hypothesis of absolute space $\mathrm{H}(\mathrm{v})$ with center of mass velocity $\mathrm{v}$. Then $\mathrm{TN}+\mathrm{H}(\mathrm{v})$ provides us with a set of infinitely many, empirically equivalent theories. Their ontological difference stems from the different nature of the velocity state of absolute space. Other examples of pairs of empirically equivalent theories are for instance: phlogiston versus oxygen theory, Ptolemaic versus Copernican cosmology, or Lorentz' ether theory versus Einstein's Special Relativity.

I maintain that in retrospect such historic cases appear mainly as artefacts of incomplete scientific knowledge - and do as such not provide really worrisome TUD cases. For we must of course expect conflicts between rivalling models at the unfinished frontier of the scientific endeavour of each age. And quite obviously the same holds in our present time as well. So the real interesting and challenging case would be the case of a serious 
underdetermination of prominent established and mature theories of our present foundational science. Are there any convincing cases like that?

\subsection{TUD examples in our actual, mature science?}

In order to meet Earman's challenge and try to find at least half-convincing cases in our actual, mature science, I will exclusively concentrate on the foundations of modern physics. My claim is that the three major branches of modern physics, namely quantum mechanics, General Relativity and gauge theories, do indeed provide us with certain TUD examples. Whether they will entirely convince us at the end of the day, is, however, another story.

The most intriguing example of TUD today, perhaps the most intriguing example ever, is in fact the case of quantum mechanics (QM) and its various ontological interpretations. While there are also interpretations that do, at least in principle, lead to different empirical consequences, there is first and foremost a whole bundle of empirically equivalent interpretations. For instance, certain variants of instrumentalistic QM, statistical (ensemble) interpretations, the Copenhagen interpretation, many worlds interpretations, consistent histories and Bohmian QM can be set up in such a way that they are, on principle grounds, empirically on a par. Spontaneous collapse theories of Ghirardi-RiminiWeber (GRW) type provide, however, an example of empirical inequivalent interpretations. Since in GRW models the collapse of the wave function is added by some additional collapse mechanism, such change of the dynamical part of the theory is of course in principle observable (it is, therefore, more appropriate to speak about GRW as a different theory rather than a QM interpretation. And due to different values of the collapse parameter we get, in fact, a whole bunch of GRW theories. ${ }^{4}$

While the case of QM is very well known, it is much less known that there do, in fact, exist various, ontologically seemingly different, yet empirically equivalent formulations of General Relativity (GR). The standard formulation of GR is given in Riemann space with the metric and the Riemann curvature tensor as basic entities. Curiously, empirically equivalent formulations can be given in terms of a gauge theory of gravity with tetrads and connections as basic entities and as a local Lorentz-invariant field theory in flat spacetime. This case not only involves an underdetermination of the particular entities, but of the mathematical structure itself. We will therefore postpone a more detailed exposition of this case to section 3, where the possibility of structural underdetermination is discussed.

A third class of TUD examples from mature physics can be found within the realm of gauge theories. I have discussed this at length elsewhere (e.g. Lyre 2004a, b) and will only

\footnotetext{
${ }^{4}$ A side-remark: GRW reminds us, again, of Duhemian holism, since it is always possible to re-establish the theory by suitable readjustments of the collapse parameter. Even worse: the readjustment procedure can be used to make GRW models auto-immune to falsification.
} 
give a short overview here. Consider the Aharonov-Bohm (AB) effect as a non-local effect quite characteristic for gauge theories: A split electron beam passes around a solenoid in which a magnetic field is confined and is brought to interference behind the solenoid. Although the region outside the solenoid is field-free, a shift in the interference pattern can be observed upon alteration of the magnetic field. It seems as if the magnetic field, the gauge field, interacts with the matter field wave function in a non-local manner, thereby violating both the idea of local action and point-like interaction. ${ }^{5}$ In order to avoid such a non-locality another, second interpretation of the effect in terms of the magnetic gauge potential can be given at the metaphysical price of giving up separability and observability of the underlying entities while at the same time retaining local action and point-like interaction. A third interpretation is also feasible in terms of holonomies (loop integrals of the potential) or Wilson loops as basic entities, which violate separability and point-like interaction, but are gauge invariant and, hence, observable. We are insofar left with three differing views about the underlying basic entities of gauge theories (fields, potentials or holonomies) and, related to this, about the validity of different metaphysical views about the precise nature of (non-) locality. The discussion of the $A B$ case, hence, provides an apparent underdetermination of the ontology of gauge theories themselves.

So what should we conclude from this? Are these convincing examples of TUD in mature science? For the moment we will accept this as an interim result. There seem to be cases where our best theories leave our knowledge about the ontology of the world underdetermined. They are not that many, but there are at least some. Perhaps we may convince ourselves at a later point that the indicated cases are not so terribly pressing, but we better postpone this discussion to the concluding section.

\section{Interim: Structural Realism and TUD}

Fortunately, for realists who feel themselves under pressure from the allegedly antirealistic force of the TUD argument, there's (at least) one option. The option is to stick with a more moderate version of scientific realism than the traditionally held full-blown entity realism, and the option goes under the heading of Structural Realism (SR). SR is roughly the view that we should be committed to the structural rather than object-like content of our mature scientific theories. Take the example of gauge theories in the preceding section. We have argued that the ontological content of modern gauge theories is underdetermined insofar as it is underdetermined by experiment whether the basic entities are gauge fields, gauge potentials or holonomies. There is, however, an important mathematical feature shared by all the rivalling views. It is given by the gauge group (in

\footnotetext{
${ }^{5}$ We should distinguish the following locality assumptions:

Point-like interaction: Interacting entities can be defined within arbitrarily small spacetime regions (usually idealized as spacetime points), couple to each other in those regions and are non-zero in overlapping regions.

Local action: All causes of an event propagate via some kind of continuous physical process.

Separability: Given a physical system S and its exhaustive, disjoint decomposition into spatiotemporally divided subsystems, it is possible to retrieve the properties of $S$ from the properties of these subsystems.
} 
this case the one-dimensional unitary group $\mathrm{U}(1))$, which figures as the central mathematical structure and by which the relevant type of theory (in this case quantum electrodynamics) is usually mainly characterised. From the SR point of view, it is this structure which should be regarded as real (Lyre 2004b). It is the structure and the entities and properties which come naturally equipped with it, if structure, and structure alone, in its most economical sense is taken into account.

Well, what is structure in general and what entities and properties come naturally equipped with it? One has to admit that the notion of the structure of a scientific theory is notoriously debatable and that the lack of a rigorous definition poses a certain problem for SR. On the other hand, the mathematical notion of structure is quite straightforward. Compare, for instance, Shapiro (2000): "Define a system to be a collection of objects with certain relations among them. [...] Define a pattern or structure to be the abstract form of the system, highlighting the interrelationships among the objects, and ignoring any features of them that do not affect how they relate to other objects in the system." From this definition it is quite clear that there are entities that come equipped with a structure, the relata, but that they are determined by structural or relational properties only. So, basically, our working definition should be that structures are domains (i.e. sets of objects) with sets of relations imposed on them.

Generally, SR comes in two flavours. Proponents of Epistemic SR (ESR) uphold the view that there are relations and relata, but that we have epistemic access to relations only. Proponents of Ontic SR (OSR) split into two groups: eliminativists and non-eliminativists about relata. Non-eliminative (or moderate) OSR is the view that there are relations and relata, but that there is nothing more to the relata than the relations in which they stand. Eliminative OSR is the view that there are only relations and no relata (well-known proponents are Steven French (2006) and James Ladyman (Ladyman and Ross 2007)). Note that under such a classification the widely debated question whether the slogan "structure is all there is" leads to the problematic position of "relations without relata" does not depend on the ESR/OSR distinction, but rather on the distinction between non-eliminative versus eliminative OSR. It is perfectly possible to uphold SR as a metaphysical position about the world without being vulnerable to the "relations without relata"-problem.

A proponent of moderate OSR is Michael Esfeld (2004). But as I've a argued elsewhere (Lyre 2009), his view is, in fact, not moderate enough, since it turns out as a natural consequence of commitment to structure that not only the relational properties but also the structural invariants as, so to speak, "structurally derived" intrinsic properties must be taken into account. The idea to individuate theories by means of their pure structural content is far too weak. There are of course lots of cases where distinct physical theories show basically the same structural content, unless we do not qualify the structure's domain. Here are some physics examples of such "structural equivalents": (i) classical electrodynamics and hydrodynamics are based on more or less the same mathematical apparatus about unspecified 'currents' including continuity equations, theorems of Gauss and Stokes etc.; (ii) the gauge theories of strong and weak isospin are both based on $\mathrm{SU}(2)$; 
(iii) the group $\mathrm{U}(1)$ figures in quantum physics both as the group of temporal automorphisms and as the gauge group of QED. Which particular structural invariants we are dealing with - whether it is electrical current or currents of certain fluids - must be decided by connecting the structural invariants with observational phenomena and cannot be decided from the pure structural theory content alone (i.e. from content which pertains the relational properties of the relata only).

I shall dub this more moderate non-eliminative version "Intermediate SR" (Lyre 2009). It is the view that there are relata and structurally derived properties, but that there is nothing more to the relata than the structurally derived intrinsic properties, where the structurally derived properties comprise relations and invariants of structure as structurally derived intrinsic properties. Note further that this position is still a viable SR position and does not collapse to old fashioned entity realism, since neither are we committed to essential properties nor are we committed to individuals. Structurally derived properties do not individuate objects but only object classes or domains of structure, but may nevertheless apply to lone objects. Theory models are individuated via structurally derived intrinsic properties connected with the observational phenomena constituting the data models.

\section{Structural Underdetermination}

In the first section we have discussed the pros and cons of TUD (mostly the cons, particularly the problem of missing examples). In second section we mentioned the idea to favour SR as an antidote against TUD and outlined a particular non-eliminative, intermediate version of SR. It is now time to bring the two issues, TUD and SR, together.

Indeed, can SR rightly be considered to be an antidote against the anti-realistic threat of TUD? The idea, again, is that while TUD undermines entity content, SR seems to avoid this by not committing us to the theory's entity content but to structural content only. The crucial question, however, is whether we can make sure that the structural content of theories is not underdetermined either. Perhaps there are cases of what one might call structural TUD? It will be instructive to review the three allegedly "interesting" cases of TUD in actual, mature science according to our analysis in section 1.3.

Let us start with the gauge theoretic example again. We already noticed in 1.3 that in this scenario the gauge group structure is indeed retained in all three interpretations. Whether one sticks with field strengths, potentials or holonomies, in all three cases one uses a mathematical machinery that is invariant under $U(1)$. There exists, in fact, the same underlying U(1)-principal fibre bundle structure in all three cases. In order to avoid entity underdetermination, the SR proponent will restrict his ontological commitment to the U(1) group structure and the property of an invariant electric charge. So it seems that the calculation works out: avoid TUD by commitment to structural content only. (This 
strategy is, by the way, in principle open to both OSR and ESR proponents. While OSR proponents commit themselves ontologically to structure only, ESR proponents might claim that we know that at least a particular structure, say $U(1)$, is instantiated by the domain of entities, but that one must remain agnostic about the particular nature of the entities.)

Let us consider the case of QM next. In 1.3 we mentioned the various interpretations of QM as an interesting case of TUD. But QM does not only provide cases for entity TUD due to its different interpretations, but perhaps also a case of structural TUD. It is a very wellknown fact that QM can be formulated by means of very different mathematical structures. Formulations of QM can be given in Hilbert space, in phase space, in noncommuting operator algebras, or in orthomodular lattices. Doesn't this provide an apparent case of structural TUD? The crucial question is whether there is something like a "structural core" connected with the empirical evidence of QM. If so, then no structural TUD prevails. And in the case of QM there is considerable evidence that precisely such a structural core exists in form of the non-commutative algebra of the theory. Moreover, the uniqueness of the Heisenberg commutation relations is ensured by a well-known representational theorem, the Stone-von Neumann-theorem. Indeed, SR proponents should focus on this relevant structural core in order to avoid structural TUD.

Admittedly, this is a rather hasty conclusion - and I do not claim it to be the ultimate wizdom about the issue. In Bohmian mechanics, for instance, a second dynamical equation besides the Schrödinger equation is introduced: the guiding equation governing particles on Bohmian trajectories. This is a seemingly new mathematical structure compared to the orthodox formulation. So perhaps there is really no structural core in the various formulations of QM and, after subjecting it to rigorous mathematical scrutiny, this turns out as a proper structural TUD case already. However, I will not delve into this issue here, because, as I will argue in a moment, the case of GR does indeed provide a better and less controversial example of structural TUD. And pointing out at least one proper case is enough for the purposes of this paper.

As mentioned in 1.3 already, the case of GR is of double interest. It seems to provide an example of both entity and structural TUD in actual, mature science. The following exposition shall be rather informal, mathematical details can be found in Lyre and Eynck (2003). Consider first the standard formulation of GR in curved Riemann space. The dynamical entity of the theory, the solution of the Einstein field equations, is the metric tensor $g_{\mu v}(x)$. The gravitational field strength is encoded in the dynamical curvature of the Riemann space, represented by the Riemann curvature tensor $R^{\mu}{ }_{v \lambda \rho}(x)$ which is basically built from the second derivatives of the metric. Gupta and Thirring in the 50's reformulated GR so as to make it look like a theory in a flat, non-dynamical background spacetime. The trick is to expand the metric in terms of a power series in the GR coupling constant $\kappa: g_{\mu v}(x)=\eta_{\mu v}(x)+\kappa h_{\mu v}(x)$, where $\eta_{\mu v}(x)$ is the flat Minkowski metric and $h_{\mu v}(x)$ is a dynamical field. By going to high enough orders in $\kappa$, flat GR can approximate standard GR to any desired accuracy (despite, perhaps, certain global topological effects). At least 
under the view of a local field theory, standard and flat GR are empirically equivalent, but while standard GR considers spacetime as dynamical and curved, flat GR assumes the world geometry to be flat and passes the dynamics to a new field entity $h_{\mu v}(x)$.

Some might complain that this is merely a "conventionalism-borderline" case not very much different from the Hollow earth example (compare section 1.2). Let us therefore go one step further and consider two formulations of GR in the framework of a gauge theory. Here, the reader should note that standard GR already includes essential features of a gauge theory, particularly a Levi-Civita connection \{\} (built from first derivatives of the metric and with Christoffel symbols \{\}$\left._{\nu \lambda}(\mathrm{x})\right)$. In gauge theoretic terms, the Christoffels \{\}$^{\mu_{\nu \lambda}}$ play the role of the gravitational gauge potentials, while the curvature tensor $\mathrm{R}^{\mu}{ }_{v \rho}$ provides the gravitational field strength. They comprise the kinematic part of the gauge Lagrangian, whereas the dynamic part is based on considering tetrads rather than the metric as basic dynamic entities which describe the matter fields in spacetime. A tetrad $\theta^{\alpha} \mu(x)$ can be pictured as a local frame of reference mediating between the spatio-temporal index $\mu$ that coordinatizes the manifold and local coordinates $\alpha$ such that the metric can be rewritten as $g_{\mu v}(x)=\eta_{\mu v}(x) \theta^{\alpha} \mu(x) \theta^{\beta_{v}}(x)$. Details aside, the replacement of the usual derivative by a covariant derivative (which includes the connection) in the dynamical equation for the tetradial matter fields, the geodesic equation, corresponds to the procedure of local gauging in GR, which is thus uncovered as possessing the structure of a proper gauge theory.

The crucial question now is what the appropriate gauge group is. A natural choice, in fact the choice which was considered first by Utiyama in the 50's, would be to reconstrue GR as a gauge theory of the homogeneous Lorentz group $\mathrm{SO}(1,3)$. It is now a remarkable feature of gravitational gauge theories that the choice of the kinetic term for the gauge fields and the corresponding gauge group is far less restricted than in the usual Yang-Mills theories. It has convincingly been argued in the literature that both a gauge theory of $\mathrm{SO}(1,3)$ and of the four-dimensional translation group $\mathrm{R}(1,3)$, a so-called translational gauge theory, are in fact empirically equivalent to standard GR (the debate has not entirely been settled, but "almost"; cf. Hayashi and Shirafuji 1979, Nester 1988). Curiously, in this theory one does not end up with a curved Riemann space but rather a teleparallel Weitzenböck space, where the gravitational field strength is represented by the torsion instead of the Riemann curvature tensor. Under this premise, a considerable ontological underdetermination occurs. Not only is the true geometrical curvature underdetermined, as in the case of standard versus flat GR (and in related conventionalism-borderline cases), but the nature of geometry itself, whether it is curvature or torsion, is indetermined as well. And even worse: The core mathematical structure itself, the gauge group, is different in both formulations of gauge GR. Structure doesn't seem to be unique. Apparently, the different gauge approaches of gravity as opposed to standard GR do not only provide a case for entity, but for structural TUD. 


\section{Conclusion: Mathematical Overdetermination, SR and TUD again}

Are there any options for SR proponents to avoid cases of structural TUD? Notice that structural TUD actually interferes with another problem, which in the terminology of Erhard Scheibe (1994) might rightly be called the "mathematical overdetermination of physics" (MOD), or, similarly, in the terminology of Michael Redhead (2002) the problem of "surplus structure". Roughly speaking, the point is that usually the mathematical parts of a physical theory exceed the physical parts - the parts that possess a proper physical interpretation. This is very often and particularly the case with exactly those mathematical formulations which seem to be best suited for application. And the puzzle, of course, is that it turns out to be very difficult, if not impossible, to eliminate such excess structure from physical theory.

Obviously, SR proponents would throw out the baby with the bath water if they just commit themselves to any mathematical theory structure whatsoever. In order to avoid the problem of MOD, SR proponents must restrict their account to what we informally already called the structural mathematical core. And it is therefore a crucial task for SR to come up with a rigorous and clear-cut definition of what can rightly be called the structural core. Unfortunately, such a general definition is not yet in sight. If it were, SR could perhaps manage to pave the way between the Scylla of structural TUD and the Charybdis of MOD.

The most prominent example of MOD in modern physics is gauge structure. And here our analysis has brought to light both a case of entity as well as a case of structural TUD in our actual and mature science. One might speculate that on a deeper understanding of gauge structure as surplus structure it is perhaps possible to overpower both scenarios. This would mean that, in particular for the latter case of structural TUD in GR, we must be able to identify the structural core of the theory of gravity with some structure beyond the gauge symmetry group structure, such that the gauge group structure itself turns out as surplus. Given our present mathematical understanding of gauge theories, the prospects of such a speculation are not very high. I conclude that, on the face of it, structural TUD is possible and is a real threat for any variant of SR.

I see, however, quite another way to undermine the force of all of the above analysed TUD scenarios. Initially, it was our interest to cope with Earman's challenge of missing TUD examples. Did we succeed? We have analysed certain cases of apparent TUD scenarios in actual, mature science. First and foremost, however, the reader should notice that the list of examples we found is still ridiculously small given the generality of the TUD thesis. And this is even more so the case if we restrict ourselves to alleged cases of structural TUD.

As I see things our small list makes it implausible that the problem of missing TUD examples is a mere "shortcoming of the philosophical literature" (pace Earman). It rather strongly undermines the plausibility of TUD itself! The small number of at least half- 
convincing TUD cases in our actual and mature science raises the suspicion that such cases might rather be considered artefacts of incomplete scientific knowledge - as we have seen them so often in the history of science. We are all familiar, for instance, with the deep and unresolved conceptual problems of quantum mechanics, in particular the measurement problem. Should we, thus, regard the various interpretations as full-blown TUD scenarios or isn't it far more plausible to assume that quantum physics itself is an unfinished and not yet settled science? And this seems even more so true in the case of GR. GR is a limited theory with a restriction on gravitational phenomena, presumably an effective theory of an underlying more fundamental theory of the structure of space and time.

From such a point of view, all of the above TUD scenarios of actual science should perhaps rather be considered "practical" cases but not cases of TUD in principle. "Practical TUD" occurs and must be expected in a science where deep and unresolved conceptual problems are still lurking in the back. As such, cases of practical TUD may rather be considered as cases of incomplete knowledge in our present science. They are not the strong anti-realistic and unavoidable horror scenarios of any future "system of the world" as Quine had them in mind, but may in fact even be utilized in a positive and constructive sense as warning indicators of open scientific problems (Lyre and Eynck 2003). This would be my second conclusion from the analyses in this paper: TUD with its few alleged and never entirely convincing cases is generally not such a terribly serious anti-realistic argument. I admit that this final conclusion requires a more thorough argumentation than I could offer here. The main task of the paper was to present and discuss at least half-convincing cases of TUD in actual science. That I did, but I doubt that they can be considered as fully convincing. To those, who do not share my doubts, I recommend to face Earman's challenge and come up with a more impressing list of cases.

\section{Acknowledgements}

Many thanks to an anonymous referee for valuable suggestions and to Gerhard Schurz and Ioannis Votsis for organizing an inspiring workshop and special issue. 


\section{References}

Earman, J. (1993). Underdetermination, Realism, and Reason. Midwest Studies in Philosophy XVIII: 19-38.

Earman, J., C. Glymour and J. Stachel, eds. (1977). Foundations of Space-Time Theories. Vol. 8, Minnesota Studies in the Philosophy of Science. University of Minnesota Press, Minneapolis.

Esfeld, M. (2004). Quantum entanglement and a metaphysics of relations. Studies in History and Philosophy of Modern Physics 35(4): 601-617.

Fraassen, B. C. van (1980). The Scientific Image. Clarendon Press, Oxford.

French, S. (2006). Structure as a Weapon of the Realist. Proceedings of the Aristotelian Society 106: 167-85.

Glymour, C. (1977). Indistinguishable Space-Times and the Fundamental Group. In: Earman et al. (1977).

Hayashi, K. and T. Shirafuji (1979). New General Relativity. Physical Review D 19 (12): 3524-3553.

Hoefer, C. and A. Rosenberg (1994). Empirical Equivalence, Underdetermination, and Systems of the World. Philosophy of Science 61: 592-607.

Laudan, L. and J. Leplin (1991). Empirical Equivalence and Underdetermination. Journal of Philosophy 88, 449-472.

Ladyman, J. and D. Ross (with D. Spurrett and J. Collier) (2007). Every Thing Must Go: Metaphysics Naturalised. Oxford University Press, Oxford.

Lyre, H. and T. O. Eynck (2003). Curve It, Gauge It, or Leave It? Underdetermination in Gravitational Theories. Journal for General Philosophy of Science 34(2): 277-303.

Lyre, H. (2004a). Lokale Symmetrien und Wirklichkeit. Mentis, Paderborn.

Lyre, H. (2004b). Holism and Structuralism in U(1) Gauge Theory. Studies in History and Philosophy of Modern Physics 35(4): 643-670.

Lyre, H. (2009). Structural Realism and Abductive-Transcendental Arguments. In: M. Bitbol, P. Kerszberg and J. Petitot (eds.): Constituting Objectivity. Transcendental Perspectives on Modern Physics. Springer, Berlin. 
Malament, D. (1977). Observationally Indistinguishable Space-Times. In: Earman et al. (1977).

Nester, J. M. (1988). Is there Really a Problem with the Teleparallel Theory? Classical and Quantum Gravity 5: 1003-1010.

Quine, W. V. (1975). On Empirically Equivalent Systems of the World. Erkenntnis 9, 313328.

Quine, W. V. (1990). Three Indeterminacies. In: R. B. Barrett and R. F. Gibson (eds.): Perspectives on Quine. Blackwell, Oxford.

Scheibe, E. (1994). On the mathematical overdetermination of physics. In: E. Rudolph and I.-O. Stamatescu (eds.): Philosophy, Mathematics and Modern Physics. Springer, Berlin, p. 186199. (Reprinted in E. Scheibe 2001: Between Rationalism and Empiricism: Selected Papers in the Philosophy of Physics, No. VIII.38. Springer, Berlin.)

Shapiro, S. (2000). Thinking about Mathematics. Oxford University Press, Oxford.

Redhead, M. (2002). The Interpretation of Gauge Symmetry. In: M. Kuhlmann, H. Lyre and A. Wayne, (eds.): Ontological Aspects of Quantum Field Theory. World Scientific, Singapore, p. 281-301. 